\title{
New amidines from intramolecular cyclization in triflic acid of nitroketene aminals with a tethered phenyl ring
}

\author{
SORO YAYA ${ }^{\mathrm{a}, *}$ BAMBA FANTÉ, ${ }^{\mathrm{b}}$ SIAKA SORHO,${ }^{\mathrm{a}}$ COUSTARD JEAN-MARIE ${ }^{\mathrm{b}}$ and \\ ADIMA A AUGUSTIN ${ }^{\mathrm{a}}$ \\ ${ }^{a}$ Laboratoire des Procédés Industriels de Synthèse et d'Environnement, Institut National \\ Polytechnique Félix Houphouët-Boigny, BP 991 Yamoussoukro, Côte d'Ivoire \\ bLaboratoire 'Synthèse et Réactivité des Substances Naturelles', UMR 6514, 40, Avenue du \\ Recteur Pineau, F-86022 Poitiers, France \\ e-mail: soro_y $@$ yahoo.fr
}

MS received 12 September 2006; revised 22 May 2007

\begin{abstract}
Nitroketene aminals with a tethered phenyl group underwent an intramolecular cyclization in trifluoromethanesulfonic acid to afford the corresponding N-(3-ethyl-hydrohydroxyiminobenzocycloalkenylidene)methylamine trifluoromethanesulfonate. The yields were fair to good excepted for the starting compound 1-[N-ethyl-N-(2-phenylethyl)amino]-1-methylamino-2-nitroethene.
\end{abstract}

Keywords. Nitroketene S,N-acetals; nitroketene aminals; trifluoromethanesulfonic acid; cyclic triflate salt; cyclization; amidine.

\section{Introduction}

The amidine functionality has been found in many natural products and amidine containing molecules are found to play a crucial role in many biological processes. ${ }^{1,2}$ The amidine moiety is an important pharmacophore in active ingredients of drugs. ${ }^{3,4}$ They are versatile building blocks for the synthesis of various heterocyclic compounds. ${ }^{5}$ In particular, aromatic amidines have been shown to function as excellent arginine side-chain mimetic due to their favorable spatial and electrostatic properties. ${ }^{6}$

Some N-Aryl substituted derivatives show insecticidal activity against the housefly, muska domestica, and special binding activity towards the nicotinic acetylcholine receptor. ${ }^{7}$ They are also of interest as naturally occurring constituents of t-RNA ${ }^{8}$ and show significant inhibition activity in oedema as well as anti-inflammatory and analgesic activities. ${ }^{9}$

Consequently, a plethora of methods has been developed for preparation of amidines ${ }^{10}$ from amides, nitriles or thioamides, and involve highly acidic, ${ }^{11}$ alkaline $^{12}$ or strongly reducing reaction conditions. ${ }^{13}$ Alternative mild methods involving the reduction of amidoximes $^{14}$ or the conversion of esters derivatives ${ }^{15}$ have also been reported.

Recently, we reported the cyclization of 1-benzylamino-1-methylsulfanyl-2-nitroethenes in triflic acid leading to formation of the corresponding diazadihydroacenaphthylene derivatives with an isoxazoline ring. ${ }^{16}$ In the present paper the synthesis of new amidines as triflate salts, starting from nitroketene aminals in trifluoromethanesulfonic acid (triflic acid) is described.

\section{Results and discussion}

\section{$2.1 \quad$ Starting material}

The starting materials 1a-d (figure 1) are unsymmetrical nitroketene aminals belonging to the 1,1 -<smiles>CCN(C)/C(=C/[N+](=O)[O-])NC</smiles>

1a-d

Figure 1. Starting materials 1a-d. 


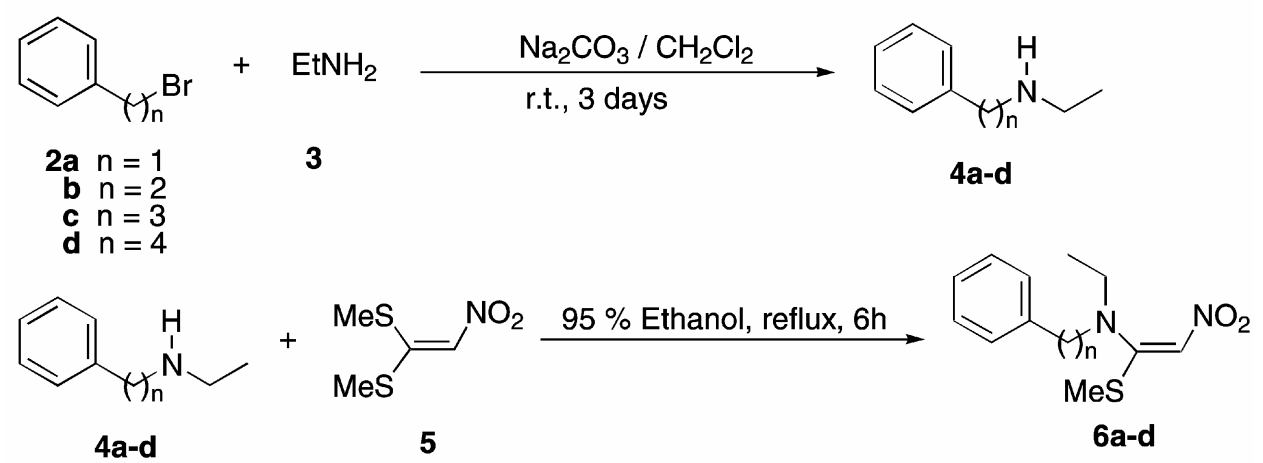

Scheme 1. Synthesis of nitroketene $S, N$-acetals 6 a-d.

Table 1. Yields of the formed nitroketene $S, N$-acetals 6a-d.

\begin{tabular}{lllll}
\hline Starting diamine & $\mathbf{4 a}$ & $\mathbf{4 b}$ & $\mathbf{4 c}$ & $\mathbf{4 d}$ \\
\hline Product & $\mathbf{6 a}$ & $\mathbf{6 b}$ & $\mathbf{6 c}$ & $\mathbf{6 d}$ \\
Yield $(\%)$ & 52 & 56 & 40 & 36 \\
\hline
\end{tabular}

bis(alkylamino)-2-nitromethylene derivatives with a tethered phenyl group on one of the nitrogen atom.

They are prepared from 1,1-bis(methylthio)-2nitroethene in a conventional way by two successive nucleophilic substitutions: ${ }^{17,18}$ the fist one, with about one molar equivalent of the corresponding $\mathrm{N}$ ethyl-N-( $\omega$-phenylalkyl)amine and, the second one, with two molar equivalents of aqueous solution of methylamine.

The N-ethyl-N-( $\omega$-phenylalkyl)amines 4 were prepared on a conventional way from the reaction of the corresponding 1-bromo- $\omega$-phenylalkyl derivative 2 with an excess of aqueous solution of ethylamine 3 (scheme 1).

In equimolar equivalent, the nucleophilic substitution between amine 4 and 1,1-bis(methylthio)-2nitroethene 5 lead to the formation of the expected nitroketene aminals $\mathbf{6}$. These reactions proceeded in yields varying from 56 to $36 \%$, decreasing with the length of the alkyl chain, probably because of entropic reason (table 1). As expected, a small amount of the di-substituted compounds was also observed.

Compounds 6 were mainly characterized by NMR and HRMS spectroscopy. The NMR spectra show thiomethyl and vinylic protons both resonating as a singulet in the range $\delta_{\mathrm{H}} 1 \cdot 15-1.19 \mathrm{ppm}$ and 6.83$6.89 \mathrm{ppm}$ respectively. The nitromethylene carbons $=\underline{\mathrm{C}} \mathrm{H}-\mathrm{NO}_{2}$ are observed at $\delta_{C} 111.7-112.9 \mathrm{ppm}$ and the $>\underline{\mathrm{C}}=\mathrm{C}$ ethylene carbons in the range $\delta_{C} 166.9$ $167.5 \mathrm{ppm}$. The thiomethyl carbons are closed to $13 \mathrm{ppm}$.

Except 6a, in organic solvent, compounds $\mathbf{6 b}-\mathbf{d}$ exist as a sole isomer as shown by a single set of signals in the ${ }^{13} \mathrm{C}$ NMR spectra. They are probably all (E)-isomers because we observed a positive NOE effect between the thiomethyl group and (i) the benzylic and (ii) the vinylic hydrogen. This NOE effect is in agreement with previously reported observations concerning the 1-arylamino-1-methylthio-2-nitroethene. $^{19}$

A further reaction between the nitroketene $S, N$ acetals $\mathbf{6}$ and two molar equivalents of aqueous solution of methylamine (40\%) afforded the nitroketene aminals 1a-d (scheme 2).

The nitroketene aminals were isolated by flash chromatography as yellow oils (eluent $\mathrm{CH}_{2} \mathrm{Cl}_{2}$ / $\mathrm{MeOH} 20: 1$ ). The yields are reported in table 2.

Compounds 1 were mainly characterized by NMR and HRMS spectroscopy. The vinylic proton resonates in the range $\delta_{H} 6.55-6.64 \mathrm{ppm}$, the nitromethylene carbon $=\underline{\mathrm{C}} \mathrm{H}-\mathrm{NO}_{2}$ and the $>\underline{\mathrm{C}}=\mathrm{C}$ ethylene carbon close to $103.8 \mathrm{ppm}$ and $163.5 \mathrm{ppm}$ respectively. These chemical shifts are at higher field than those observed for the compounds $\mathbf{6 a}-\mathbf{d}$ because of the heavy atom effect of sulphur. The methylamine protons resonate in the range $\delta_{H} 2 \cdot 88-3 \cdot 06 \mathrm{ppm}$ as a doublet because of a coupling with the amine proton $\left({ }^{3} J_{\mathrm{HH}} 5 \cdot 0-5 \cdot 3 \mathrm{~Hz}\right)$ whereas their carbon resonates close to $32.6 \mathrm{ppm}$.

In organic solvent, these compounds exist as a sole isomer as shown by a single set of signals in the ${ }^{13} \mathrm{C}$ NMR spectra. They are probably all $(E)$-isomers because this conformation allows the formation of an intramolecular hydrogen bond between the $\mathrm{N}-\mathrm{H}$ and one of the oxygen of the $-\mathrm{NO}_{2}$ groups, as previ- 


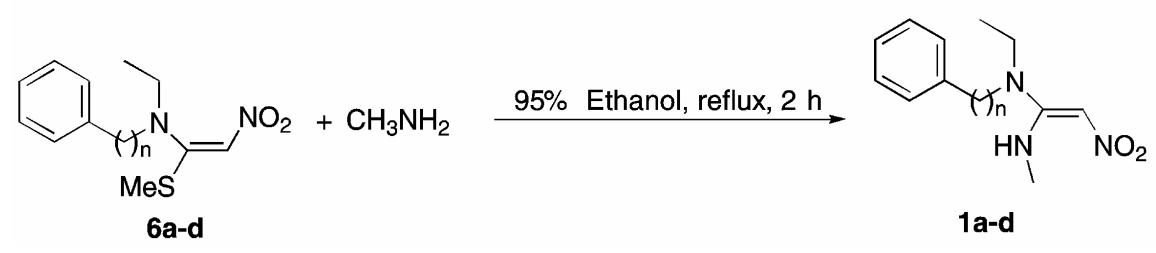

Scheme 2. Synthesis of nitroketene aminals 1a-d.

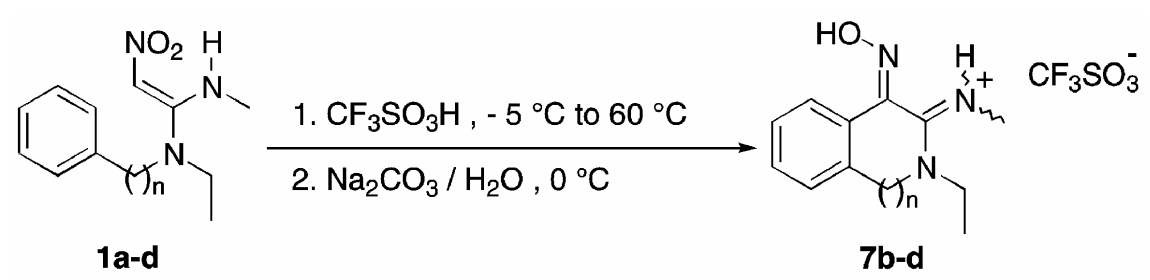

Scheme 3. Synthesis of amidinium trifluoromethanesulfonates $\mathbf{7 b}-\mathbf{d}$.

Table 2. Yields of nitroketene aminals 1a-d.

\begin{tabular}{lllll}
\hline $\begin{array}{l}\text { Starting nitroketene } \\
S, N \text {-acetals }\end{array}$ & $\mathbf{6 a}$ & $\mathbf{6 b}$ & $\mathbf{6 c}$ & $\mathbf{6 d}$ \\
\hline Product & $\mathbf{1 a}$ & $\mathbf{1 b}$ & $\mathbf{1 c}$ & $\mathbf{1 d}$ \\
Yield (\%) & 52 & 56 & 40 & 36 \\
\hline
\end{tabular}

ously reported for the 1-arylamino-1-methylthio-2nitroethenes. ${ }^{19}$

\subsection{Reactions in trifluoromethanesulfonic acid}

The nitroketene aminals $\mathbf{1 a - d}$ dissolved easily in triflic acid. The molar ratio trifluoromethanesulfonic $\mathrm{acid} /$ nitroketene aminal was $50: 1$. The reaction was carried out at $60^{\circ} \mathrm{C}$ under nitrogen atmosphere (scheme 3).

The starting material was fully transformed after $24 \mathrm{~h}$. At the end of the reaction and after cooling, the solution was poured into $50 \mathrm{~mL}$ of $\mathrm{CH}_{2} \mathrm{Cl}_{2} /$ $\mathrm{MeOH}(95: 5)$ at $-20^{\circ} \mathrm{C}$. Thereafter, the resulting solution was poured over ice ( $15 \mathrm{~g})$ and anhydrous $\mathrm{Na}_{2} \mathrm{CO}_{3}(6.0 \mathrm{~g} ; 56.6 \mathrm{mmol})$. The aqueous phase was quickly extracted with $\mathrm{CH}_{2} \mathrm{Cl}_{2} / \mathrm{MeOH}(95: 5)$. The reaction was generally clean with yields varying from 43 to $93 \%$ (table 3 ) excepted for 1 a because of degradation reactions leading to the formation of dark polar products not otherwise studied.

The structures of compounds $\mathbf{7 b}-\mathbf{d}$ were determined by NMR and HRMS spectroscopy. The $=\mathrm{N}-\mathrm{H}$ proton appeared as a broad singlet in the range of 9.24-9.29 ppm and the $=\mathrm{N}-\mathrm{OH}$ in the range of $12 \cdot 24-12 \cdot 37 \mathrm{ppm}$. The $\mathrm{C}=\mathrm{N}-\mathrm{H}$ and $\mathrm{C}=\mathrm{N}-\mathrm{OH}$ carbons resonate at $\delta_{C} \quad 146 \cdot 1-147 \cdot 1 \mathrm{ppm}$ and $161 \cdot 1-$ $162 \cdot 1 \mathrm{ppm}$ respectively.

The formation of compounds $\mathbf{7 b}-\mathbf{d}$ may be explained by a similar mechanism as reported for acyclic nitroketene $S, S$-acetals ${ }^{20}$ (scheme 4).

In this mechanism, the starting products $\mathbf{1}$ undergo a protonation on the carbon bearing the nitro group and an O-protonation of the nitro group. This last $\mathrm{O}$ protonation occurred through a fast proton exchange process with the acidic medium, as demonstrated in fluorosulfonic acid at $-80^{\circ} \mathrm{C}^{21}$ Prototropic exchanges and the formal loss of a molecule of water lead to the formation of the reacting conjugated hydroxynitrilium ion $8{ }^{20}$ The formation of dications $\mathbf{8}$ occurs through a rate limiting step that needs heating at $60^{\circ} \mathrm{C}$, probably because the water elimination needs a further protonation to occur. In agreement with this hypothesis is the fact that with the less basic sulphur atom, formation of the stable hydroxynitrilium ions occurred even at low temperature. ${ }^{20,22}$ As soon as it is formed, the hydroxynitrilium ion 8 reacts with the tethered phenyl ring to afford the cyclic stable doubly protonated hydroxyiminoamidine 9 .

At the end of the reaction, when the acidity was destroyed, compounds $\mathbf{7 b} \mathbf{b}-\mathbf{d}$ were isolated as triflate salts because of the strong basicity of the amidine group. $^{23}$

\section{Experimental section}

\subsection{General remarks}

Melting points were determined with a Büchi Melting point B545 apparatus using capillary tubes (tem- 


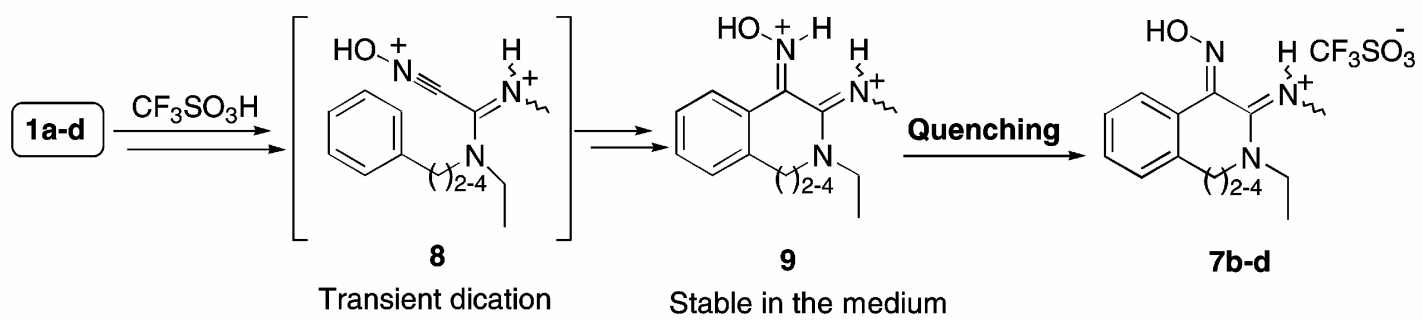

Scheme 4. Suggested mechanism for the formation of amidinium trifluoromethanesulfonates 7.

Table 3. Yields of amidine triflate salts $7 \mathbf{a}-\mathbf{d}$.

\begin{tabular}{lcccc}
\hline Starting compound & 1a & 1b & 1c & 1d \\
\hline Amidine triflate salt & $\mathbf{7 a}$ & $\mathbf{7 b}$ & $\mathbf{7 c}$ & $\mathbf{7 d}$ \\
$\begin{array}{l}\text { Yield (\%) in recovered } \\
\text { product }\end{array}$ & Trace & 62 & 93 & 43 \\
\hline
\end{tabular}

perature rate $2^{\circ} \mathrm{C} / \mathrm{mn}$ ) and were not corrected. - A Brucker DPX 300 spectrometer, equipped with a low temperature probe, was used for ${ }^{1} \mathrm{H}-,{ }^{9} \mathrm{~F}$ - and ${ }^{13} \mathrm{C}-\mathrm{NMR}$ spectra recorded at $300 \cdot 13,282.37$ and $75.47 \mathrm{MHz}$, respectively. NMR spectra were recorded at room temperature and chemical shifts reported relative to $\mathrm{Me}_{4} \mathrm{Si}$ or $\mathrm{CFCl}_{3}$ for fluorine. The reproducibility of ${ }^{13} \mathrm{C}$ NMR shift was about $\pm 0.05 \mathrm{ppm}$, depending on cell and concentration. Chemical assignments were made using DEPT and homo/hetero 2D techniques' and/or usual chemical shift assignments rules. - High Resolution Mass Spectrometry was performed by the 'Centre Régional de Mesures Physiques de l'Ouest-Université de Rennes, France'. - Flash chromatography was achieved on silica gel (20 to $45 \mu \mathrm{m}$ particle size). - Trifluoromethanesulfonic acid was purchased from Across and 1,1-bis(methylthio)-2-nitroethene from Lancaster and were used without further purification. No special attempt was made to optimise the yields.

\subsection{1-[N-(benzyl)ethylamino]-1-methylthio-2- nitroethene (6a). - Typical procedure}

1,1-bis(methylthio)-2-nitroethene (1.82 g, $11 \mathrm{mmol})$ and the $\mathrm{N}$-(benzyl)ethylamine $(1.35 \mathrm{~g}, 10 \mathrm{mmol})$ were heated together in refluxing 95\% ethanol $(75 \mathrm{~mL})$ under nitrogen atmosphere. The reaction was monitored by thin-layer chromatography $\left(\mathrm{CH}_{2} \mathrm{Cl}_{2} /\right.$ ethyl acetate : 20/1 and $\mathrm{MeOH} / \mathrm{NH}_{3}$ aq. 10/0 1). After disappearance of the $\mathrm{N}$-(benzyl)ethylamine $(\mathrm{MeOH} /$ $\mathrm{NH}_{3}$ aq.: 10/0.1) $(6 \mathrm{~h})$ the reacting medium was cooled and concentrated under reduced pressure.
The resulting oil was purified by flash chromatography (eluent $\mathrm{CH}_{2} \mathrm{Cl}_{2}$ /ethyl acetate: 20/1) to afford the oily nitroethene derivative $6 \mathbf{a}(1.31 \mathrm{~g}, 52 \%)$ as a mixture of $(\mathrm{Z}) /(\mathrm{E})$ isomers in about $1 / 10$ ratio. $-R_{f}=$ $0.4\left(\mathrm{CH}_{2} \mathrm{Cl}_{2}\right.$ /ethyl acetate $\left.\left.20: 1\right)\right)-{ }^{1} \mathrm{H} \mathrm{NMR}\left(\mathrm{CDCl}_{3}\right.$, * major isomer): $\delta_{\mathrm{H}}=1.19$ and $1.24^{*}(t, 3 \mathrm{H}$, $\left.J=6.9 \mathrm{~Hz}, \mathrm{CH}_{2}-\mathrm{CH}_{3}\right), 2.54$ and $2.54^{*}(\mathrm{~s}, 3 \mathrm{H}$, $\left.\mathrm{CH}_{3} \mathrm{~S}\right), 3 \cdot 56^{*}$ and $4.12\left(q, 2 \mathrm{H}, \mathrm{J}=7 \cdot 0 \mathrm{~Hz}, \mathrm{CH}_{2}-\mathrm{Me}\right)$, 4.65 and $4.72 *\left(s, 2 \mathrm{H}, \mathrm{CH}_{2}-\mathrm{Ph}\right), 6.60$ and $6.89^{*}(s$, $1 \mathrm{H}$, vinylic $\mathrm{H}), 7 \cdot 18$ and $7 \cdot 18^{*}(d, J=6.5 \mathrm{~Hz}, 2 \mathrm{H}$, ar. $o-\mathrm{H}), 7 \cdot 2-7.4$ and $7 \cdot 2 *-7.4 *(m, 3 \mathrm{H}$, ar. $o-$ and $m-\mathrm{H}) .-{ }^{13} \mathrm{C}$ NMR $\left(\mathrm{CDCl}_{3}, *\right.$ major isomer $)$ : $\delta_{\mathrm{C}}=13.6^{*}$ and $14.6\left(\mathrm{CH}_{2}-\mathrm{CH}_{3}\right), 18.3^{*}$ and 21.4 $\left(\mathrm{SCH}_{3}\right), 48 \cdot 5^{*}$ and 51.0 $\left(\mathrm{CH}_{2}-\mathrm{Me}\right), 57 \cdot 0^{*}$ and 60.8 $\left(\mathrm{CH}_{2}-\mathrm{Ph}\right), 112.9$ and $112.9 *\left(=\mathrm{CH}-\mathrm{NO}_{2}\right), 127.5$ and $127.5^{*}$ (ar. CH), 128.5 and $128.5^{*}$ (ar. CH), 129.4 and $129.4^{*}$ (ar. CH), 135.5 and $135.5^{*}$ (ipso-C), $167.4 *$ and $171.5 \quad(>\mathrm{N}-\mathrm{C}=) .-\mathrm{HRMS}$ for $\mathrm{C}_{12} \mathrm{H}_{16} \mathrm{~N}_{2} \mathrm{O}_{2} \mathrm{~S}[M]^{+}$: calcd. $225 \cdot 1517$, found $225 \cdot 1519$ and for $\mathrm{C}_{11} \mathrm{H}_{13} \mathrm{~N}_{2} \mathrm{O}_{2} \mathrm{~S}\left[\mathrm{M}-\mathrm{CH}_{3}\right]^{+}$: calcd. 210.1283, found $210 \cdot 1287$.

\subsection{1-(N-ethyl-2-phenylethylamino)-1- (methylthio)-2-nitroethene (6b)}

From N-ethyl-2-phenylethylamine (1.49 g, $10 \mathrm{mmol})$ and 1,1-bis(methylthio)-2-nitroethene (1.82 g, $11 \mathrm{mmol})$ was obtained the expected nitro derivative 6b (1.49 $\mathrm{g}, 56 \%)$ as a yellow oil. $-R_{f}=0.3\left(\mathrm{CH}_{2} \mathrm{Cl}_{2} /\right.$ ethyl acetate $(20: 1))-{ }^{1} \mathrm{H} \mathrm{NMR}\left(\mathrm{CDCl}_{3}\right): \delta_{\mathrm{H}}=1.22$ $\left(t, 3 \mathrm{H}, J=7.2 \mathrm{~Hz}, \mathrm{CH}_{2}-\mathrm{CH}_{3}\right), 2.40\left(s, 3 \mathrm{H}, \mathrm{CH}_{3} \mathrm{~S}\right)$, $2.92\left(c t, 2 \mathrm{H}, J=7.7 \mathrm{~Hz}, \mathrm{CH}_{2}-\mathrm{Ph}\right), 3.52(q, 2 \mathrm{H}$, $\left.J=7.2 \mathrm{~Hz}, \mathrm{CH}_{2}-\mathrm{Me}\right), 3.76\left(c t, 2 \mathrm{H}, J=7.7 \mathrm{~Hz}, \mathrm{CH}_{2}-\right.$ $\mathrm{N}<), 6.87(s, 1 \mathrm{H}$, vinylic $\mathrm{H}), 7.17-7.36(m, 5 \mathrm{H}$, ar. $\mathrm{H}) . \quad{ }^{13} \mathrm{C} \mathrm{NMR}\left(\mathrm{CDCl}_{3}\right): \delta_{\mathrm{C}}=13.5\left(\mathrm{CH}_{3}\right), 18.3$ $\left(\mathrm{CH}_{3}\right), 34.8\left(\mathrm{CH}_{2}-\mathrm{Ph}\right), 49 \cdot 2\left(\mathrm{CH}_{2}-\mathrm{Me}\right), 54 \cdot 7\left(\mathrm{CH}_{2}-\right.$ $\mathrm{N}), 112.4\left(=\mathrm{CH}-\mathrm{NO}_{2}\right), 127.3$ (ar. CH), 129.1 (ar. $\mathrm{CH}$ ), 129.2 (ar. CH), 137.8 (ipso-C), 166.9 (-NH$\mathrm{C}=)$. $-\mathrm{HRMS}$ for $\mathrm{C}_{13} \mathrm{H}_{18} \mathrm{NOS}[\mathrm{M}-\mathrm{NO}]^{+}$: calcd. 236.1109, found 236.1124. 
3.4 1-(N-ethyl-3-phenylpropylamino)-1(methylthio)-2-nitroethene (6c)

From N-ethyl-3-phenylpropylamine $(1.63 \mathrm{~g}, 10 \mathrm{mmol})$ and 1,1-bis(methylthio)-2-nitroethene $\quad(1.82 \mathrm{~g}$, $11 \mathrm{mmol}$ ) was obtained the expected nitro derivative 6c $(1 \cdot 12 \mathrm{~g}, 40 \%)$ as a yellow oil. $-R_{f}=0.2\left(\mathrm{CH}_{2} \mathrm{Cl}_{2} /\right.$ ethyl acetate $(30: 1))-{ }^{1} \mathrm{H} \mathrm{NMR}\left(\mathrm{CDCl}_{3}\right): \delta_{\mathrm{H}}=1 \cdot 20$ $\left(t, 3 \mathrm{H}, J=7.2 \mathrm{~Hz}, \mathrm{CH}_{3}\right), 1.97\left(m, 2 \mathrm{H}, \mathrm{CH}_{2}\right), 2.47(s$, $\left.3 \mathrm{H}, \mathrm{CH}_{3} \mathrm{~S}\right), 2.67\left(t, 2 \mathrm{H}, J=7.6 \mathrm{~Hz}, \mathrm{CH}_{2}-\mathrm{Ph}\right), 3.53$ $\left(m, 4 \mathrm{H}, \mathrm{CH}_{2}\right), 6 \cdot 83(\mathrm{~s}, 1 \mathrm{H}$, vinylic $\mathrm{H}), 7 \cdot 15-7.25(m$, $3 \mathrm{H}$, ar. $o-\mathrm{H}$ and $p-\mathrm{H}), 7.28(m, 2 \mathrm{H}$, ar. $m-\mathrm{H}) .-{ }^{13} \mathrm{C}$ NMR $\left(\mathrm{CDCl}_{3}\right): \delta_{\mathrm{C}}=13.6\left(\mathrm{CH}_{3}\right), 18 \cdot 3\left(\mathrm{SCH}_{3}\right), 29.9$ $\left(\mathrm{CH}_{2}\right), 33 \cdot 1\left(\mathrm{CH}_{2}-\mathrm{Ph}\right), 49 \cdot 0\left(\mathrm{CH}_{2}-\mathrm{Me}\right), 52.7\left(\mathrm{CH}_{2}-\right.$ $\mathrm{N}), 111.9\left(=\mathrm{CH}-\mathrm{NO}_{2}\right), 126.7($ ar. $\mathrm{CH}), 128.7$ (ar. $\mathrm{CH}), 129.0$ (ar. CH), 140.9 (ipso-C), $167 \cdot 3$ (-NH$\mathrm{C}=)$. - HRMS for $\mathrm{C}_{14} \mathrm{H}_{20} \mathrm{NOS}[\mathrm{M}-\mathrm{NO}]^{+}$: calcd. $250 \cdot 1265$, found $250 \cdot 1257$. HRMS for $\mathrm{C}_{14} \mathrm{H}_{19} \mathrm{NS}$ $\left[\mathrm{M}-\mathrm{NO}_{2} \mathrm{H}\right]^{+}$: calcd. 233.1238, found 233.1244.

\subsection{1-(N-ethyl-4-phenylbutylamino) -1- (methylthio)-2-nitroethene (6d)}

From N-ethyl-4-phenylbutylamine $(1.77 \mathrm{~g}, 10 \mathrm{mmol})$ and 1,1-bis(methylthio)-2-nitroethene (1.82 g, $11 \mathrm{mmol})$ was obtained the expected nitro derivative $\mathbf{6 d}$ $(1.06 \mathrm{~g}, 36 \%)$ as a yellow oil. $R_{f}=0.34\left(\mathrm{CH}_{2} \mathrm{Cl}_{2} /\right.$ ethyl acetate $(30: 1))-{ }^{1} \mathrm{H}$ NMR $\left(\mathrm{CDCl}_{3}\right): \delta_{\mathrm{H}}=1.21$ $\left(t, 3 \mathrm{H}, J=7.2 \mathrm{~Hz}, \mathrm{CH}_{3}\right), 1.65\left(m, 4 \mathrm{H}, 2 \mathrm{CH}_{2}\right), 2.48$ $\left(s, 3 \mathrm{H}, \mathrm{CH}_{3} \mathrm{~S}\right), 2.65\left(\mathrm{~m}, 2 \mathrm{H}, \mathrm{CH}_{2}-\mathrm{Ph}\right), 3.50(\mathrm{ct}, 2 \mathrm{H}$, $\left.J=7.2 \mathrm{~Hz}, 2 \mathrm{CH}_{2}\right), 3.52\left(q, 2 \mathrm{H}, J=7.2 \mathrm{~Hz}, \mathrm{CH}_{2-}\right.$ $\mathrm{Me}), 6.83(\mathrm{~s}, 1 \mathrm{H}$, vinylic $\mathrm{H}), 7 \cdot 15-7.25(\mathrm{~m}, 3 \mathrm{H}$, ar. $\mathrm{H}), 7.27-7.33(\mathrm{~m}, 2 \mathrm{H}$, ar. $\mathrm{H}) .-{ }^{13} \mathrm{C} \mathrm{NMR}\left(\mathrm{CDCl}_{3}\right)$ : $\delta_{\mathrm{C}}=13.6 \quad\left(\mathrm{CH}_{3}\right), \quad 18.8 \quad\left(\mathrm{SCH}_{3}\right), 27.9 \quad\left(\mathrm{CH}_{2}\right), 28.7$ $\left(\mathrm{CH}_{2}\right), 35.8\left(\mathrm{CH}_{2}-\mathrm{Ph}\right), 48.8\left(\mathrm{CH}_{2}-\mathrm{Me}\right), 53.3\left(\mathrm{CH}_{2}-\right.$ $\mathrm{N}), 111.7\left(=\mathrm{CH}-\mathrm{NO}_{2}\right), 126.4($ ar. $\mathrm{CH}), 128.75($ ar. $\mathrm{CH}), 128.83$ (ar. $\mathrm{CH}$ ), 141.9 (ipso-C), 167.4 (-NH$\mathrm{C}=)$. - HRMS for $\mathrm{C}_{15} \mathrm{H}_{22} \mathrm{NOS}\left([\mathrm{M}-\mathrm{NO}]^{+}\right)$: calcd. 264.1422, found 264.1407. HRMS for $\mathrm{C}_{15} \mathrm{H}_{22} \mathrm{NS}$ $\left[\mathrm{M}-\mathrm{NO}_{2}\right]^{+}$: calcd. 248.1473, found 248.1466. HRMS for $\mathrm{C}_{14} \mathrm{H}_{19} \mathrm{~N}_{2} \mathrm{O}_{2}$ [M-SMe] $]^{+}$: calcd. 247.1447, found $247 \cdot 1455$.

\subsection{1-[N-(benzyl)ethylamino]-1-(methylamino)-2-} nitroethene (1a). - Typical procedure

1-[N-(benzyl)ethylamino]-1-(methylthio)-2-nitroethene 6a $(756 \mathrm{mg}, 3 \mathrm{mmol})$ and the aqueous solution of methylamine $(5.2 \mathrm{~mL}, 6 \mathrm{mmol})$ were heated together in refluxing $95 \%$ ethanol $(25 \mathrm{~mL})$ under nitrogen. The reaction was monitored by thin-layer chromatography $\left(\mathrm{CH}_{2} \mathrm{Cl}_{2} / \mathrm{MeOH}: 20 / 1\right)$. After disappear- ance of the starting compound $6 \mathbf{a}(2 \mathrm{~h})$ and cooling, the mixture was concentrated under reduced pressure and the resulting oily product was purified by flash chromatography (eluent $\mathrm{CH}_{2} \mathrm{Cl}_{2} / \mathrm{MeOH}(20: 1)$ to afford compound $1 \mathrm{a}(548 \mathrm{mg}, 78 \%)$ as a yellow oil. $-R_{f}=0.54\left(\mathrm{CH}_{2} \mathrm{Cl}_{2} / \mathrm{MeOH}(20: 1)\right)-{ }^{1} \mathrm{H}$ NMR $\left(\mathrm{CDCl}_{3}\right): \delta_{\mathrm{H}}=1 \cdot 19\left(t, 3 \mathrm{H}, J=7 \cdot 0 \mathrm{~Hz}, \mathrm{CH}_{2}-\mathrm{CH}_{3}\right)$, $3.06\left(d, 3 \mathrm{H}, J=5.0 \mathrm{~Hz}, \mathrm{CH}_{3}-\mathrm{NH}\right), 3.19(t, 1 \mathrm{H}$, $\left.J=7.0 \mathrm{~Hz}, \mathrm{CH}_{2}-\mathrm{Me}\right), 4.41\left(\mathrm{~s}, 2 \mathrm{H}, \mathrm{CH}_{2}-\mathrm{Ph}\right), 6.60(\mathrm{~s}$, $1 \mathrm{H}$, vinylic $\mathrm{H}), 7.22(d, 2 \mathrm{H}, J=7.2 \mathrm{~Hz}$, ar. $o-\mathrm{H})$, 7.3-7.4 ( $m, 3 \mathrm{H}$, ar. $p$ - and $m-\mathrm{H}), 9.9$ (broad $s, \mathrm{NH})$. $-{ }^{13} \mathrm{C}$ NMR $\left(\mathrm{CDCl}_{3}\right): \delta_{\mathrm{C}}=12.8\left(\mathrm{CH}_{3}\right), 32.7\left(\mathrm{CH}_{3} \mathrm{~N}\right)$, $44.7\left(\mathrm{CH}_{2}-\mathrm{Me}\right), 53.4\left(\mathrm{CH}_{2}-\mathrm{Ph}\right), 103.7\left(=\mathrm{CH}-\mathrm{NO}_{2}\right)$, 127.6 (ar. $\mathrm{CH}), 128.4$ (ar. $\mathrm{CH}), 129.4$ (ar. $\mathrm{CH})$, 135.8 (ipso-C), 163.6 (-NEt-C=). - HRMS for $\mathrm{C}_{12} \mathrm{H}_{17} \mathrm{~N}_{3} \mathrm{O}_{2}[M]^{+}$: calcd. 235.1321, found 235.1322. HRMS for $\mathrm{C}_{12} \mathrm{H}_{17} \mathrm{~N}_{2}$ [M-NO $\left.\mathrm{NO}_{2}\right]^{+}$: calcd. 189.1392, found $189 \cdot 1398$.

\subsection{1-(N-ethyl-2-phenylethylamino)-1- (methylamino)-2-nitroethene (1)}

From 1-(N-ethyl-2-phenylethylamino)-1-(Methylthio)-2-nitroethene $\mathbf{6 b}(798 \mathrm{mg}, 3 \mathrm{mmol})$ and the aqueous solution of methylamine $(5.2 \mathrm{~mL}, 6 \mathrm{mmol})$ was obtained the expected nitroethene derivative $\mathbf{1 b}$ (714 mg, 96\%) as yellow oil. $-R_{f}=0.54\left(\mathrm{CH}_{2} \mathrm{Cl}_{2} /\right.$ $\mathrm{MeOH}(20: 1))-{ }^{1} \mathrm{H}$ NMR $\left(\mathrm{CDCl}_{3}\right): \delta_{\mathrm{H}}=1.15(t$, $\left.3 \mathrm{H}, \quad J=7.1 \mathrm{~Hz}, \quad-\mathrm{CH}_{2}-\mathrm{CH}_{3}\right), \quad 2.88 \quad(t, 2 \mathrm{H}, \quad J=$ $\left.7.31 \mathrm{~Hz}, \mathrm{CH}_{2}\right), 2.89\left(d, 3 \mathrm{H}, J=5.3 \mathrm{~Hz},-\mathrm{HN}-\mathrm{CH}_{3}\right)$, $3.44\left(d d, 2 \mathrm{H}, J=7.2 \mathrm{~Hz}, J=6.1 \mathrm{~Hz}, \mathrm{CH}_{2}\right), 6.57(s$, $1 \mathrm{H}$, vinylic $\mathrm{H}), 7 \cdot 14-7 \cdot 18(m, 2 \mathrm{H}$, ar. $\mathrm{H}), 7 \cdot 2-7 \cdot 35$ $(m, 3 \mathrm{H}$, ar. $\mathrm{H}), 9.9$ (broad $s, \mathrm{NH}),-{ }^{13} \mathrm{C}$ NMR $\left(\mathrm{CDCl}_{3}\right): \delta_{\mathrm{C}}=13 \cdot 1\left(\mathrm{CH}_{3}\right), 32.6\left(\mathrm{CH}_{3} \mathrm{~N}\right), 34.2\left(\mathrm{CH}_{2}-\right.$ $\mathrm{Ph}), 45.8\left(\mathrm{CH}_{2}-\mathrm{Me}\right), 49.3\left(\mathrm{CH}_{2}\right), 50.9\left(\mathrm{CH}_{2}-\mathrm{N}\right)$, $103.9\left(=\mathrm{CH}-\mathrm{NO}_{2}\right), 127.3($ ar. $\mathrm{CH}), 129.0($ ar $\mathrm{CH})$, $127 \cdot 3$ (ar. $\mathrm{CH}), 138 \cdot 1$ (ipso-C), $163 \cdot 2$ (-NEt-C=). HRMS for $\mathrm{C}_{13} \mathrm{H}_{19} \mathrm{~N}_{2} \mathrm{O}$ [M-NO] ${ }^{+}$: calcd. 219.1497, found $219 \cdot 1494$.

\subsection{1-(N-ethyl-3-phenylpropylamino)-1- (methylamino)-2-nitroethene (1c)}

From 1-(N-ethyl-3-phenylpropylamino)-1-(methylthio)-2-nitroethene $6 \mathbf{c}(840 \mathrm{mg}, 3 \mathrm{mmol})$ and the aqueous solution of methylamine $(5.2 \mathrm{~mL}, 6 \mathrm{mmol})$ was obtained the expected nitroethene derivative 1c (747 mg, 95\%) as a yellow oil. $-R_{f}=0.35\left(\mathrm{CH}_{2} \mathrm{Cl}_{2} /\right.$ $\mathrm{MeOH} 20: 1) .-{ }^{1} \mathrm{H}$ NMR $\left(\mathrm{CDCl}_{3}\right): \delta_{\mathrm{H}}=1.15(t, 3 \mathrm{H}$, $\left.J=7.0 \mathrm{~Hz}, \mathrm{CH}_{3}\right), 1.92(m, 2 \mathrm{H}, J=7.3 \mathrm{~Hz}, J=7.5 \mathrm{~Hz}$, $\left.\mathrm{CH}_{2}\right), 2.62\left(t, 2 \mathrm{H}, J=7.3 \mathrm{~Hz}, \mathrm{CH}_{2}-\mathrm{Ph}\right), 2.90(d, 3 \mathrm{H}$, $\left.J=5 \cdot 1 \mathrm{~Hz}, \mathrm{CH}_{3}-\mathrm{N}\right), 3 \cdot 17\left(t, 2 \mathrm{H}, J=7 \cdot 8 \mathrm{~Hz}, \mathrm{CH}_{2}-\right.$ 
$\mathrm{N}<), 3.23\left(q, J=7.0 \mathrm{~Hz}, \mathrm{CH}_{2}-\mathrm{Me}\right), 6.55(s, 1 \mathrm{H}$, vinylic $\mathrm{H}), 7.15(d, 2 \mathrm{H}, J=7.2 \mathrm{~Hz}$, ar. $o-\mathrm{H}), 7.26(d$, $1 \mathrm{H}, J=6.9 \mathrm{~Hz}$, ar. $p-\mathrm{H}), 7.30(d d, 2 \mathrm{H}, J=6.9 \mathrm{~Hz}$, $J=7.2 \mathrm{~Hz}$, ar. $m-\mathrm{H}), 9.91$ (broad $s, \mathrm{NH}) .-{ }^{13} \mathrm{C}$ NMR $\left(\mathrm{CDCl}_{3}\right): \delta_{\mathrm{C}}=12.8\left(\mathrm{CH}_{3}\right), 29.0\left(\mathrm{CH}_{2}\right), 32.2$ $\left(\mathrm{CH}_{3} \mathrm{~N}\right), \quad 32.8 \quad\left(\mathrm{CH}_{2}-\mathrm{Ph}\right), \quad 44.8 \quad\left(\mathrm{CH}_{2}-\mathrm{Me}\right), \quad 48.7$ $\left(\mathrm{CH}_{2} \mathrm{~N}\right), 103.3\left(=\mathrm{CH}-\mathrm{NO}_{2}\right), 126 \cdot 3($ ar. $\mathrm{CH}), 128 \cdot 3$ (ar. $\mathrm{CH}), 128.6$ (ar. $\mathrm{CH}), 140.4$ (ipso-C), 163.0 (-NEt-C $=$ ). - HRMS for $\mathrm{C}_{14} \mathrm{H}_{21} \mathrm{~N}_{3} \mathrm{O}_{2}[M]^{+}$: calcd. 263.1633, found 263.1613. HRMS for $\mathrm{C}_{14} \mathrm{H}_{21} \mathrm{~N}_{2}$ [M$\left.\mathrm{NO}_{2}\right]^{+}$: calcd. 217.1704, found 217.1701.

\subsection{1-(N-ethyl-4-phenylbutylamino)-1-}

(methylamino)-2-nitroethene (1d)

From 1-( $N$-ethyl-4-phenylbutylamino)-1-(Methylthio)-2-nitroethene $6 \mathrm{~d}(882 \mathrm{mg}, 3 \mathrm{mmol})$ and the aqueous solution of methylamine $(5 \cdot 2 \mathrm{~mL}, 6 \mathrm{mmol})$ was obtained the expected nitroethene derivative 1d (737 $\mathrm{mg}, 89 \%)$ as a yellow oil. $-R_{f}=0.36\left(\mathrm{CH}_{2} \mathrm{Cl}_{2} /\right.$ $\mathrm{MeOH} 20: 1) .-{ }^{1} \mathrm{H} \mathrm{NMR}\left(\mathrm{CDCl}_{3}\right): \delta_{\mathrm{H}}=1.16(t, 3 \mathrm{H}$, $\left.J=7.1 \mathrm{~Hz}, \mathrm{CH}_{3}\right), 1.61\left(m, 2 \mathrm{H}, \mathrm{CH}_{2}\right), 2.63(m, 2 \mathrm{H}$, $\left.\mathrm{CH}_{2}-\mathrm{Ph}\right), 2.97\left(d, 3 \mathrm{H}, J=5.3 \mathrm{~Hz}, \mathrm{CH}_{3} \mathrm{~N}\right), 3.17(m$, $\left.2 \mathrm{H}, \mathrm{CH}_{2} \mathrm{Me}\right), 3.22\left(q, J=7 \cdot 1 \mathrm{~Hz}, 2 \mathrm{H}, \mathrm{CH}_{3}-\mathrm{CH}_{2}-\mathrm{N}\right)$, $6.64(s, 1 \mathrm{H}$, vinylic $\mathrm{H}), 7.18(m, 3 \mathrm{H}$, ar. $o-$ and $p-\mathrm{H})$, $7.28(m, 2 \mathrm{H}$, ar. $m-\mathrm{H}), 9.94$ (broad $s, \mathrm{NH}) .-{ }^{13} \mathrm{C}$ NMR $\left(\mathrm{CDCl}_{3}\right): \delta_{\mathrm{C}}=13.2\left(\mathrm{CH}_{3}\right), 27 \cdot 3\left(\mathrm{CH}_{2}\right), 28.9$ $\left(\mathrm{CH}_{2}\right), 32 \cdot 7\left(\mathrm{CH}_{3} \mathrm{~N}\right), 35 \cdot 8\left(\mathrm{CH}_{2}-\mathrm{Ph}\right), 45 \cdot 1\left(\mathrm{CH}_{2}-\mathrm{Me}\right)$, $49.6\left(\mathrm{CH}_{2}-\mathrm{N}\right), 103.7\left(=\mathrm{CH}-\mathrm{NO}_{2}\right), 126.4($ ar. $\mathrm{CH})$, 128.7 (ar. CH), 128.9 (ar. CH), 141.9 (ipso-C), $163.4(-\mathrm{NEt}-\mathrm{C}=)$ ). - HRMS for $\mathrm{C}_{15} \mathrm{H}_{23} \mathrm{~N}_{3} \mathrm{O}_{2}[M]^{+}$: calcd. 277.1790, found 277.1795.

3.10 (Z)-N-[3-ethyl-1,2-dihydro-5-hydroxyimino$1 \mathrm{H}$-benzo[d]azepin-4(3H)-ylidene]methylamine trifluoromethanesulfonate (7b) - Typical procedure

1-(N-ethyl-2-phenylethylamino)-1-(Methylamino)2-nitroethene 1 b (248 $\mathrm{mg}, 1 \mathrm{mmol})$ was dissolved in trifluoromethanesulfonic acid $(4.4 \mathrm{~mL}, 50 \mathrm{mmol})$ at $-5^{\circ} \mathrm{C}$. The solution was then heated to $60^{\circ} \mathrm{C}$ for $24 \mathrm{~h}$. After cooling the solution was poured into $50 \mathrm{~mL}$ of a cool $\left(-20^{\circ} \mathrm{C}\right)$ mixture of dichloromethane/methanol $(95: 5)$ and the resulting mixture was then poured over ice $(15 \mathrm{~g})$ and anhydrous $\mathrm{Na}_{2} \mathrm{CO}_{3}$ $(6.0 \mathrm{~g} ; 56.6 \mathrm{mmol})$. The aqueous phase was quickly extracted with $\mathrm{CH}_{2} \mathrm{Cl}_{2} / \mathrm{MeOH}(95: 5)(4 \times 50 \mathrm{~mL})$. The organic layers were dried over $\mathrm{MgSO}_{4}$ and the solvent evaporated under reduced pressure. The resulting product was purified by flash chromatography with $\mathrm{CH}_{2} \mathrm{Cl}_{2} / \mathrm{MeOH}(10: 1)$ to afford the triflate salt $7 \mathrm{~b}(236 \mathrm{mg}, 62 \%)$ as white crystals. $-R_{f}=0.3$
$\left(\mathrm{CH}_{2} \mathrm{Cl}_{2} / \mathrm{MeOH}(20: 1)\right)$. - m.p. $191 \cdot 0^{\circ} \mathrm{C}\left(\mathrm{CH}_{2} \mathrm{Cl}_{2} /\right.$ $\mathrm{MeOH}$ (95:5)/petroleum ether). $\quad-{ }^{1} \mathrm{H}$ NMR ([D $\left.D_{6}\right]$ acetone) $: \delta_{\mathrm{H}}=1.37\left(t, 3 \mathrm{H}, J=7.2 \mathrm{~Hz}, \mathrm{CH}_{3}\right)$, $3.21\left(s, 3 \mathrm{H}, \mathrm{CH}_{3}-\mathrm{N}\right), 3 \cdot 28(m, 1 \mathrm{H}$, benzylic $\mathrm{H}), 3.43$ (complex $t, 1 \mathrm{H}, J \approx 16 \mathrm{~Hz}$, benzylic $\mathrm{H}), 3.84(\mathrm{~m}, 2 \mathrm{H}$, $\left.\mathrm{CH}_{2}-\mathrm{Me}\right), 3.92(m, 1 \mathrm{H}$, cyclic $\mathrm{N}-\mathrm{CH}<), 4.34(t$, $J=14 \mathrm{~Hz}, 1 \mathrm{H}$, cyclic $\mathrm{N}-\mathrm{CH}<), 7.37(m, 2 \mathrm{H}$, ar. $\mathrm{H})$, $7.47(m, 1 \mathrm{H}$, ar. H), 7.78 (complex $d, J=8 \mathrm{~Hz}, 1 \mathrm{H}$, ar. $\mathrm{H}), 8.8$ (very broad $\left.s, 1 \mathrm{H},=\mathrm{N}^{+} \mathrm{H}-\mathrm{Me}\right), 12 \cdot 2$ (very broad $s, 1 \mathrm{H},>=\mathrm{N}-\mathrm{OH}) .-{ }^{13} \mathrm{C}$ NMR $\left(\left[D_{6}\right]\right.$ acetone): $\delta_{\mathrm{C}}=12.6\left(\mathrm{~N}-\mathrm{CH}_{2}-\mathrm{CH}_{3}\right), 32.6\left(\mathrm{CH}_{2}-\mathrm{Ph}\right), 33.0\left(\mathrm{CH}_{3}-\right.$ $\left.{ }^{+} \mathrm{NH}=\right), 46 \cdot 2\left(\mathrm{~N}-\mathrm{CH}_{2}-\mathrm{Me}\right), 50 \cdot 5\left(\right.$ cyclic $\left.-\mathrm{CH}_{2}-\mathrm{N}\right)$, $122 \cdot 4\left(q, J=318 \mathrm{~Hz}, \mathrm{CF}_{3}-\mathrm{SO}_{3}{ }^{-}\right.$), $126 \cdot 2$ (ipso-C), 127.4 (ar. $\mathrm{CH}), 132.0$ (ar. $\mathrm{CH}), 132.1$ (ar. $\mathrm{CH})$, 132.2 (ar. $\mathrm{CH}), 137.6($ ipso-C), $146 \cdot 1(>\mathrm{C}=\mathrm{N}-\mathrm{H})$, $162 \cdot 1(>\mathrm{C}=\mathrm{N}-\mathrm{OH}) .-{ }^{19} \mathrm{~F}$ NMR $\left(\left[D_{6}\right]\right.$ acetone $): \delta=$ $-78.9\left(\mathrm{CF}_{3} \mathrm{SO}_{3}^{-}\right)$. $-\mathrm{MS}(70 \mathrm{eV}) ; \mathrm{m} / \mathrm{z}(\%): 232$ $\left[\mathrm{M}-\mathrm{CF}_{3} \mathrm{SO}_{3}\right]^{+}$. $-\mathrm{HRMS}$ for $\mathrm{C}_{13} \mathrm{H}_{18} \mathrm{~N}_{3} \mathrm{O}\left[\mathrm{M}-\mathrm{CF}_{3} \mathrm{SO}_{3}\right]^{+}$: calcd. $232 \cdot 1450$, found $232 \cdot 1443$.

3.11 (Z)-N-[3-ethyl-3,4,5,6-tetrahydro-1-hydroxyiminobenzo[d]azocin-2(lH)-ylidene]methylamine trifluoromethanesulfonate (7c)

From 1-(N-ethyl-3-phenylpropylamino)-1-(Methylamino)-2-nitroethene $1 \mathrm{c}(262 \mathrm{mg}, 1 \mathrm{mmol})$ was obtained the cyclic triflate salt 7c $(368 \mathrm{mg}, 93 \%)$ as white crystals. $-R_{f}=0.2\left(\mathrm{CH}_{2} \mathrm{Cl}_{2} / \mathrm{MeOH}(20: 1)\right)$. m.p. $214 \cdot 1{ }^{\circ} \mathrm{C}\left(\mathrm{CH}_{2} \mathrm{Cl}_{2} /\right.$ petroleum ether $) .-{ }^{1} \mathrm{H} \mathrm{NMR}$ ([D $\left.D_{6}\right]$ acetone): $\delta_{\mathrm{H}}=1.42\left(t, 3 \mathrm{H}, J=7.2 \mathrm{~Hz}, \mathrm{CH}_{2}-\right.$ $\left.\mathrm{CH}_{3}\right), 2.24\left(m, 2 \mathrm{H}, \mathrm{CH}_{2}\right), 2.93\left(m, 2 \mathrm{H}, \mathrm{CH}_{2}\right), 3.20(\mathrm{~s}$, $\left.3 \mathrm{H}, \mathrm{N}-\mathrm{CH}_{3}\right), 3.83\left(q, 2 \mathrm{H}, \mathrm{CH}_{2}-\mathrm{CH}_{3}\right), 4.08(t, 2 \mathrm{H}$, $\left.J=6.5 \mathrm{~Hz},-\mathrm{CH}_{2}-\mathrm{N}<\right), 7.40-7.55(m, 3 \mathrm{H}$, ar. $\mathrm{H})$, $8.16(d d, 1 \mathrm{H}, J=3.2 \mathrm{~Hz}, J=2.0 \mathrm{~Hz}$, ar. $\mathrm{H}), 9.0(v b s$, $1 \mathrm{H},>=\mathrm{NH}), 12 \cdot 3(\mathrm{vbs}, 1 \mathrm{H},>=\mathrm{N}-\mathrm{OH}) .-{ }^{13} \mathrm{C}$ NMR ([D $\left.D_{6}\right]$ acetone): $\delta_{\mathrm{C}}=11.9\left(\mathrm{CH}_{3}\right), 29.6\left(\mathrm{CH}_{2}\right), \quad 32.7$ $\left(\mathrm{NH}-\mathrm{CH}_{3}\right), 32.9\left(\mathrm{CH}_{2}-\right), 46 \cdot 3\left(\mathrm{CH}_{2}-\mathrm{Me}\right), 52.4\left(\mathrm{CH}_{2}-\right.$ $\mathrm{N}<), 122 \cdot 3\left(q, J_{\mathrm{CF}}=318 \mathrm{~Hz}, \mathrm{CF}_{3} \mathrm{SO}_{3}^{-}\right), 128 \cdot 0$ (ar. $\mathrm{CH}), 128.9$ (ipso-C), 132.48 (ar. $\mathrm{CH}), 132.52$ (ar. $\mathrm{CH}), 133.3$ (ar. CH), 141.1 (ipso-C), $146.9(>=\mathrm{N}-$ $\mathrm{H}), 161.8(>=\mathrm{N}-\mathrm{OH}) . \quad{ }^{19} \mathrm{~F}$ NMR $\left(\left[D_{6}\right]\right.$ acetone $)$ : $\delta=-78.9\left(\mathrm{CF}_{3} \mathrm{SO}_{3}^{-}\right) .-\mathrm{MS}(70 \mathrm{eV}) ; \mathrm{m} / z(\%): 246$ $\left[\mathrm{M}-\mathrm{CF}_{3} \mathrm{SO}_{3}\right]^{+}$. - HRMS for $\mathrm{C}_{14} \mathrm{H}_{20} \mathrm{~N}_{3} \mathrm{O}\left[\mathrm{M}-\mathrm{CF}_{3} \mathrm{SO}_{3}\right]^{+}$: calcd. 246 1606 , found $246 \cdot 1613$.

\subsection{2 (Z)-N-[3-ethyl-4,5, 6,7-tetrahydro-1-} hydroxyimino- $1 \mathrm{H}$-benzo[d]azonin-2(3H)-ylidene] methylamine trifluoromethanesulfonate (7d)

From 1-(N-ethyl-4-phenylbutylamino)-1-(Methylamino)-2-nitroethene $1 \mathbf{d}(276 \mathrm{mg}, 1 \mathrm{mmol})$ was obtained the cyclic triflate salt $\mathbf{7 d}(177 \mathrm{mg}, 43 \%)$ as 
white crystals. $-R_{f}=0.4\left(\mathrm{CH}_{2} \mathrm{Cl}_{2} / \mathrm{MeOH}(10: 1)\right)$. m.p. $147 \cdot 6^{\circ} \mathrm{C}$ (acetone/petroleum ether). $-{ }^{1} \mathrm{H}$ NMR ([D $\left.D_{6}\right]$ acetone): $\delta_{\mathrm{H}}=1.32\left(t, 3 \mathrm{H}, J=7.2 \mathrm{~Hz}, \mathrm{CH}_{3}\right)$, $1.73\left(\right.$ bs $, 2 \mathrm{H}, \mathrm{CH}_{2}$ ), 2.06 (quintuplet, $2 \mathrm{H}, \mathrm{CH}_{2}$ ), 2.88 $\left(t, 2 \mathrm{H}, J=6 \mathrm{~Hz}, \mathrm{CH}_{2}\right), 3.26\left(b s, 3 \mathrm{H}, \mathrm{CH}_{3}-\mathrm{N}<\right), 3.74$ (quadruplet, $\left.2 \mathrm{H}, J=7.2 \mathrm{~Hz}, \mathrm{CH}_{2} \mathrm{Me}\right), 3.78(b s, 2 \mathrm{H}$, $\left.-\mathrm{CH}_{2}-\mathrm{N}\right), 7 \cdot 3-7.5(m, 4 \mathrm{H}$, ar. $\mathrm{H}), 9 \cdot 1(v b s, 1 \mathrm{H}$, $>=\mathrm{NH}), \quad 12 \cdot 2 \quad(v b s, 1 \mathrm{H},>=\mathrm{N}-\mathrm{OH}) . \quad-{ }^{13} \mathrm{C}$ NMR ([D $]$ acetone): $\delta_{\mathrm{C}}=11.9\left(\mathrm{CH}_{3}\right), 29.1\left(\mathrm{CH}_{2}\right), 31.6(b s$, $\left.\mathrm{CH}_{2}\right), 33.6\left(\mathrm{CH}_{3}-\mathrm{N}\right), 47.4 \quad\left(\mathrm{CH}_{2}-\mathrm{Me}\right), 51.5 \quad(\mathrm{bs}$, $\left.-\mathrm{CH}_{2}-\mathrm{N}<\right), 122 \cdot 3\left(q, J_{\mathrm{CF}}=318 \mathrm{~Hz}, \mathrm{CF}_{3} \mathrm{SO}_{3}{ }^{-}\right), 128 \cdot 0$ (ar. CH), 129.6 (bs, ar. CH), 130.5 (bs, ipso-C), 132.0 (ar. CH), 132.4 (ar. CH), 142.4 (ipso-C), $147 \cdot 1 \quad(>=\mathrm{N}-\mathrm{H}), \quad 161 \cdot 1 \quad(>=\mathrm{N}-\mathrm{OH}) . \quad-{ }^{19} \mathrm{~F}$ NMR ([D $]$ acetone): $\delta=-78.9\left(\mathrm{CF}_{3} \mathrm{SO}_{3}{ }^{-}\right) .-\mathrm{MS}(70 \mathrm{eV})$; $m / z$ (\%): $260\left[\mathrm{M}-\mathrm{CF}_{3} \mathrm{SO}_{3}\right]^{+}$. $-\mathrm{HRMS}$ for $\mathrm{C}_{15} \mathrm{H}_{22} \mathrm{~N}_{3} \mathrm{O}$ ([M-CF $\left.\mathrm{SO}_{3}\right]^{+}$): calcd. 260.1763, found 260.1754.

\section{Conclusion}

In the present study, the bicyclic N-(3-ethylhydrohydroxyiminobenzocycloalkenylidene) methylamine trifluoromethanesulfonates salts have been easily prepared from various 1-[N-ethyl-N-( $\omega$-phenylalkyl) amino]-1-methylamino-2-nitroethenes in trifluoromethanesulfonic acid. Theses derivatives may be used in natural products synthesis and work in this field is in progress.

\section{Acknowledgement}

Thanks to CNRS for the financial support and to 'Ministère des Affaires Étrangères' for fellowship fund.

\section{References}

1. Naka M, Nanbu T, Kobayashi K, Kamanaka Y, Komeno M, Yanasa R, Fukutomi T, Fujimura S, Seo N G, Fujiwara N, Ohuchida S, Suzuki K, Kondo K and Taniguchi N 2000 Biochem. Biophys. Res. Commun. 270663

2. Tucker J A, Clyton T L, Chidester C G, Schulz M W, Harrington L E, Conrad S J, Yagi Y, Oien N L, Yurek D and Kuo 2000 Bioorg. Med. Chem. 8601
3. Buschi C A and Pomilio A B 1987 Phytochemistry 26 863

4. De la Rosa R, Martinez-Barraza V, Burgos C and Alvarez-Builla J 2000 Tetrahedron Lett. 415837

5. Boyd G V 1991 Reactions and synthetic uses of amidines, in the chemistry of amidines and imidates (eds) S Patai and Z Rappoport (New York: John Wiley) vol 2, pp 367-424

6. (a) Zablocki J A, Miyano M, Garland R B, Pireh D, Schretzman L, Rao S N, Lindmark R J, PanzerKnodle S G, Nicholson N S, Taite B B, Salyers A K, King L W, Campion J G and Feigen L P 1993 J. Med. Chem. 36 1811; (b) Peterlin-Mašič L and Kikelj D 2001 Tetrahedron 577073

7. Nishiwaki H, Nakagowa Y, Ueno T, Kagabu S and Mishimura K 2001 Pest Manage. Sci. 57810

8. Bailey C, Baku E, Hayler J and Kane P 1999 Tetrahedron Lett. $\mathbf{4 0} 4847$

9. Stahle H 2000 Best Practice Res. Clin. Anaesthesiol. 14237

10. (a) Gauter J A, Miocque M and Farnoux C C 1975 In The chemistry of amidines and imidates (ed.) S Patai (New York: John Wiley) vol. 1, pp 283-348; (b) Boyd G V 1991 In The chemistry of amidines and imidates (eds) S Patai and Z Rappoport (New York: John Wiley) vol. 2, pp 367-424

11. Stilz H U, Jablonka B, Just M, Knoll J, Paulus E F and Zoller G 1996 J. Med. Chem. 392118

12. Thurkauf A and Laballed J 1997 Compd. Radiopharm. 39123

13. Judkins B D, Allen D G, Cook T A, Evans B and Sardharwala T A 1996 Synth. Commun. 264351

14. Cesar J, Nadrah K and Dolenc M S 2004 Tetrahedron Lett. $\mathbf{4 5} 7445$

15. Gielen H, Alonso-Alija C, Hendrix M, Niewöhner U and Schauss D 2002 Tetrahedron Lett. 43419

16. Soro Y, Bamba F, Siaka S and Coustard J-M 2006 Tetrahedron Lett. 473315

17. Rajappa S 1999 Tetrahedron 557065

18. Pavlova Z F, Lipina E S and Perekalin V V 1995 Sulphur Report 16149

19. Kearney T, Harris P A, Jackson A and Joule J A 1992 Synthesis 8769

20. Coustard J-M 2001 Eur. J. Org. Chem. 1525

21. (a) Olah G A and Kiovski T E $1968 \mathrm{~J}$. Am. Chem. Soc. 90 6461; (b) Olah G A, Fung A P and Rawdah T N 1980 J. Org. Chem. 454149

22. (a) Coustard J-M 1999 Tetrahedron 55, 5809; (b) Coustard J-M 1996 Tetrahedron 5219509

23. Häfelinger G and Kuske F K H 1991 In The chemistry of amidine and imidate (eds) S Patai and Z Rappoport (New York: John Wiley) pp 1-93; and references cited therein 